\title{
Failure Rate of Orthodontic Mini-screw after Insertion using 3D Printed Guide versus Conventional Free Hand Placement Technique: Split Mouth Randomized Clinical Trial
}

\author{
Hadir Aboshady* (D), Amr Mohamed Aly Abouelezz, Mai Hamdy Aboul Fotouh, Sherif Aly Mahmoud Elkordy \\ Department of Orthodontics, Faculty of Dentistry, Cairo University, Giza, Egypt
}

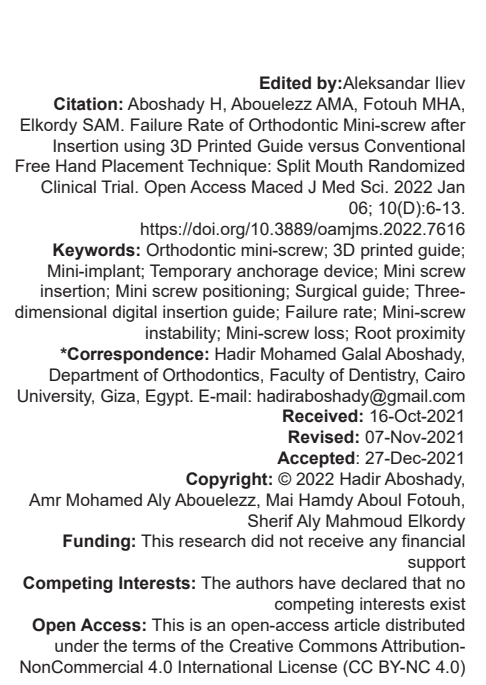

\begin{abstract}
AIM: The aim of the study is to assess the failure rate after mini-screw insertion using digital three-dimensional printed guide versus free hand placement technique through a well-designed split-mouth randomized clinical trial.

METHODS: Forty-two patients with mean age (22.56 \pm 3.47 years) indicated for upper first premolars' extraction (Bimaxillary protrusion and Class II division 1) were included in the study. Their maxillary quadrants were randomized to receive mini-screws as means of anchorage. Pre-operative maxillary cone-beam computed tomography scan with ultra-low-dose protocol was imaged and the maxillary arch was scanned using intra-oral scanner to obtain stereo-lithographic format file for the maxillary arch. Using in vivo and Rapidform Geomagic Studio ${ }^{\circledR}$ Softwares the mini-screws were planned to be inserted in the buccal inter-radicular space between the upper second premolar and first molar in both right and left sides. For the intervention sides; digital three-dimensional guides were designed and printed for mini-screw insertion. Failure of the mini-screws was assessed till 3 months of loading
\end{abstract}

RESULTS: There was no statistical significant difference in failure rate of mini-screws in both intervention $(7.14 \%)$ and control sides $(16.6 \%)$, with weak and moderate correlation between the root proximity and the mini-screws failure in intervention and control groups respectively.

CONCLUSIONS: Using a digital three-dimensional printed guide for mini-screw insertion had no effect on the failure rate of the inserted mini-screws.

REGISTRATION: ClinicalTrials.gov Identifier: NCT03653078.

\section{Introduction}

Temporary anchorage devices (TADs) had been widely used in recent orthodontics [1]. They could be considered important tools during orthodontic treatment. They have the advantage of providing efficient anchorage even if patient's compliance is an issue. In addition, TADs could facilitate appropriate biomechanics when anchorage management is critical [2].

Among the several types of TADs, mini-screws considered the most commonly used type. Mini-screws have the advantage of simplicity in application among other TADs. They could be inserted and removed without the need of surgery. Moreover, due to their reduced dimensions, they could be inserted in the inter-radicular regions in the maxilla and mandible. Various designs, lengths, diameters, and materials were proposed in the market to accommodate the increasing need of miniscrews in orthodontics which made their cost is the most convenient in comparison to other TADs. However, they have their own limitations and risks [3], [4], [5].
Mini-screw are supposed to be stable till the end of the orthodontic treatment. If the screw showed signs of looseness, mobility or inflammation that necessitated its removal or relocation, the mini-screw would be considered failed [6]. Mini-screws' failure can be attributed to multiple factors; patient-related and mini-screw related factors [7]. Patient's sex, age, bone quality, medical condition, oral hygiene, smoking habit, and craniofacial skeletal pattern could affect the stability and failure rate of the inserted mini-screws [8]. Moreover, the mini-screw's length and diameter also have a crucial influence on their success. In addition to other factors such as; the arch where the mini-screw inserted (maxilla or mandible), the site of insertion (buccal, lingual, or alveolar ridge) and the bone quality in that area [9].

Another important factor was reported in the literature was the proximity of the mini-screw to the adjacent roots, especially in the buccal inter-radicular region [10], [11], [12]. The mini-screw that was in close proximity to the roots could initiate unwanted inflammatory process around the adjacent tissues which would jeopardize the mini-screws stability [13]. 
From a different perspective, forces applied on the teeth in contact of the mini-screw could easily transmitted to the mini-screw itself [14]. Therefore, accurate and precise mini-screw placement is considered mandatory to avoid mini-screw's instability and finally failed.

Guided mini-screw insertion for individual patients was proposed in the literature in many forms. Simple wire guide combined with two-dimensional radiograph was the start point [15]. Further sophisticated designs were introduced using different materials such as acrylic resin [16] and thermoplastic materials [17]. In the recent digital era, the technology of the 3D printing paved the way to construct a simple accurate guide for mini-screw insertion. Thanks to recent technological advances, combining 3D scanning and cone-beam computed tomography (CBCT) provide complete visualization of the roots and buccal cortical bone. This allowed planning a clear unobstructed path for miniscrew insertion that can be transferred through a physical guide obtained from three-dimensional printing would allow precise mini-screw insertion with the least possible risks. Furthermore, the assessment of the mini-screw's proximity to the roots was previously performed using another CBCT scan that was taken post-operatively [18], [19], exposing the patients to another dose of radiation in quite short period. For that reason, a less invasive method is needed. Therefore, a well-designed splitmouth randomized clinical trial was performed to assess the difference between the failure rate of mini-screws inserted with 3D printed guide versus the freehand conventional method and provide insightful clinical recommendations for accurate mini-screw insertion.

\section{Methods}

\section{Trial design}

Split mouth randomized clinical trial parallel design where allocation and randomization of the eligible patients was with ratio 1:1.

\section{Sample size calculation}

Sample size calculated depending on a previous study (Hourfar et al. [20]) as reference. According to this study, the probability of exposure among controls is 0.3 . If the true probability of exposure among cases is 0.05 , we will need to study 35 patients per group to be able to reject the null hypothesis that the exposure rates for case and controls are equal with probability (power) 0.8. The Type I error probability associated with this test of this null hypothesis is 0.05 . Chi-squared test was performed using Power and sample size program. Total sample size increased to 42 subjects per group to compensate $20 \%$ drop out.

\section{Participants}

Eligibility criteria

- Adult orthodontic patients age range (18-30 years)

Orthodontic patients indicated for upper first premolars' extraction with maximum anchorage

- Non-smoker patients

- $\quad$ Patients without missing teeth in the upper arch except the third molar

- Medically free patients with no bleeding disorders, bone diseases, osteoporosis, or on anticoagulant therapy

- $\quad$ Patients with facial symmetry with no cleft lip and palate.

\section{Study settings}

The patients enrolled in the study were selected and diagnosed from the outpatient clinic, Department of Orthodontics, Faculty of Dentistry, Cairo University.

\section{Randomization}

\section{Sequence generation}

Computer-generated site [1] was used for allocation and randomization of the eligible patients with ratio $1: 1$

\section{Allocation concealment}

- $\quad$ Allocation concealment was done through opaque sealed envelopes, whereas each envelope had a code number

- $\quad$ Each code was representing the side where the intervention was applied (right/left) for each participant.

\section{Implementation}

- $\quad$ Each eligible patient received a sealed envelope, inside which a code only known to the associate supervisor

According to that code, each side of the patient was assigned for intervention or control according to the list of codes of randomization

- $\quad$ Principal investigator knew which side was the intervention or control only after planning the mini-screw position for both sides for each patient

Implementation procedure was applied just before designing the 3D guide for the intervention side. 


\section{Blinding (masking)}

- $\quad$ The current trial was single blinded

- The patients and the principal investigator couldn't be blinded as the two insertion techniques were completely different.

\section{Interventions}

\section{Pre-treatment records}

In the first clinical appointment of all enrolled patients, the following records were taken; primary impressions, full set of intra and extraoral photographs, pre-operative CBCT with ultra-low dose protocol using Planmeca ProMax® 3D Max [2] of the skull $(90 \mathrm{kV}, 7.1 \mathrm{~mA}$, $25 \mathrm{mGy} \cdot \mathrm{cm}^{2}$ ), the patients were instructed to open their mouth during the imaging procedures keeping their upper and lower teeth apart from each other and pre-operative intra-oral scan

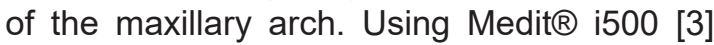
Intra-oral scanner to obtain stereo-lithographic format (STL) for mini-screw planning In addition, the mini-screw used in our study GNI Smart Anchor [4] bracket-head design with $1.6 \mathrm{~mm}$ diameter and $8 \mathrm{~mm}$ length was scanned with the same intra-oral scanner twice to obtain 2 STL files; STL of the mini-screw and the other STL consisted of the shank of the driver with the mini-screw attached to it.

\section{Planning mini-screw position}

This procedure was done for each patient for both right and left sides by the principal investigator before the implementation of the randomized codes in the sealed envelopes

Preoperative digital imaging and communications in medicine CBCT scan was installed in in vivo dental viewer to be converted into an STL file

The two STLs of the maxilla obtained from the intra-oral scanning and the CBCT scan were then installed in Rapidform Geomagic Studio $\AA$ Software [5], they were superimposed on each other using landmark-based superimposition technique. In this technique one STL used as a reference (STL of the CBCT scan) and the other used as test, then multiple identical points were selected in both STLs to be superimposed on each other

Then the STL of the mini-screw was planned to be positioned in buccal inter-radicular space midway between the upper second premolar and first molar with an angle $40^{\circ}$ with the long axis of the roots according to Lim et al. [21] in the right and left sides (Figure 1)

Afterward, the STL of the mini-screw attached to the shank of the driver was substituted

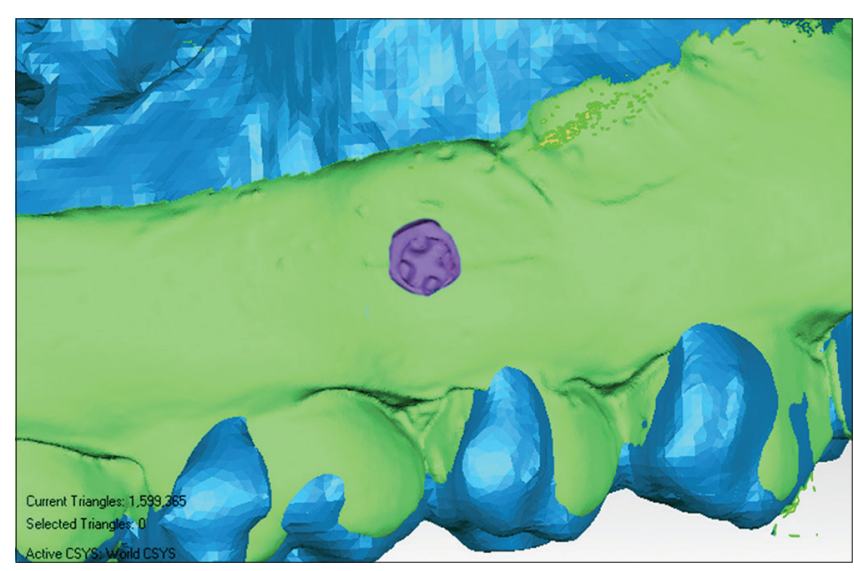

Figure 1: A window of Geomagic software showing stereolithographic format from cone beam computed tomography scan (blue) superimposed on the maxillary scan (green) and the miniscrew in the planned position (purple)

with the STL of the mini-screw alone in the intervention side.

\section{$3 D$ guide}

\section{Designing and 3D printing of the mini-screw}

- $\quad$ The STL of the maxilla with the driver in position was uploaded in 3 Shape ${ }^{\circledR}$ software [6] to design the 3D guide

- The boundaries of the guide were drawn over the maxillary arch. The guide of $4 \mathrm{~mm}$ thickness was designed to cover the occlusal surfaces of second premolar, the first molar, and half of first premolar extending palatal and buccal beyond the position of the mini-screw with a cylinder of $11 \mathrm{~mm}$ length added at the position of the mini-screw to the buccal wall with inner diameter equal to the outer diameter of the driver (Figure 2)

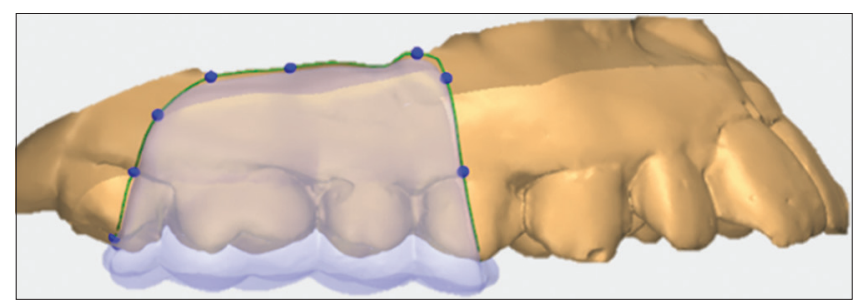

Figure 2: A window of the 3Shape software showing the buccal extension of the $3 D$ guide

Then the design was saved and printed by RasDENT® [7] 3D printer using NextDent surgical guide Class I biocompatible resin [8], finished and polished.

\section{Mini-screw insertion in patient mouth}

Before mini-screw insertion, topical anesthetic gel was applied, then few drops of local anesthetic solution [9] (Articaine 1:100000, 4\% Adrenaline) were given by infiltration in the site of the mini-screw insertion 
- Insertion site preparation was done through swapping with sterile gauze and Betadine antiseptic solution in one direction from apical to coronal

- In the intervention side, the 3D printed guide was inserted in the patient's mouth with complete seating, and the patient was instructed to bite on a cotton pellet to hold the guide in place, then the driver with the miniscrew was inserted through the channel of the guide till complete mini-screw's insertion (Figure 3)

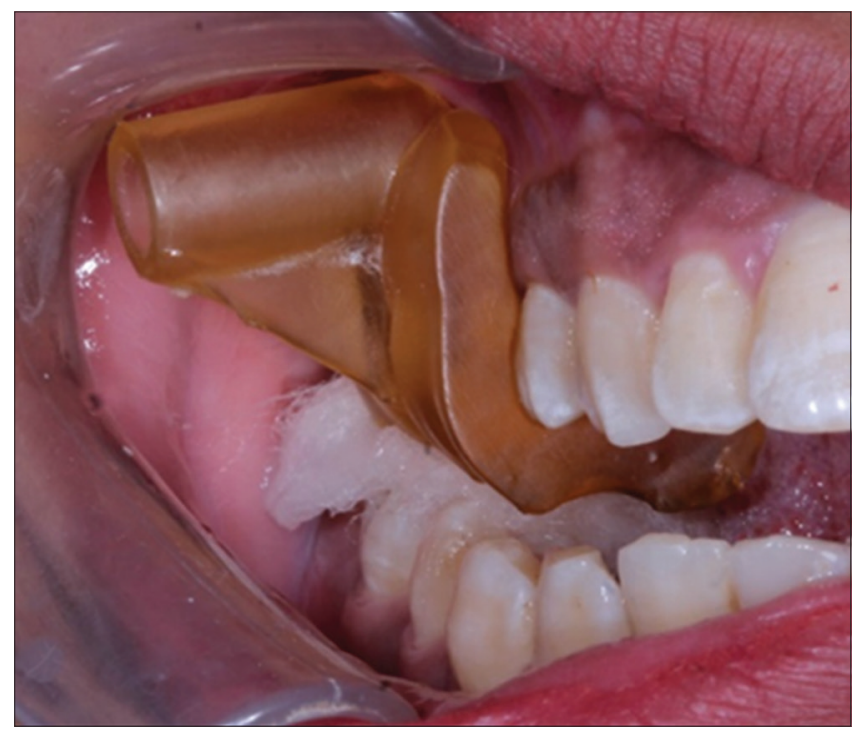

Figure 3: 3D printed guide in position, patient was biting on cotton palette to ensure full seating

- In the control side, the insertion site of miniscrew was located conventionally with the help of the CBCT scan. The explorer was pressed inter-dentally between the second premolar and first molar to create a line, at the intersection between this line and the mucogingival junction a bleeding point was created with the tip of the explorer to mark the site of insertion of the mini-screw, at which the mini-screw was inserted with an acute angle with the bone.

\section{in patient mouth}

Recording mini-screw position after insertion

- The maxillary arch including the buccally positioned mini-screws on both sides was scanned with the same intra-oral scanner to create post-insertion scan STL

- $\quad$ This STL was superimposed using the same technique on the STL of the maxilla from the CBCT scan in the Geomagic software to get the relation between the inserted mini-screw and the adjacent roots. The distance between the mini-screw and the buccal root of the second premolar was measured $\bullet$

According to that distance, the mini-screws were categorized into two groups; group A where this distance was $\leq 0.6 \mathrm{~mm}$, group $B$ where this distance was $\geq 0.6 \mathrm{~mm}$ (Figure 4).

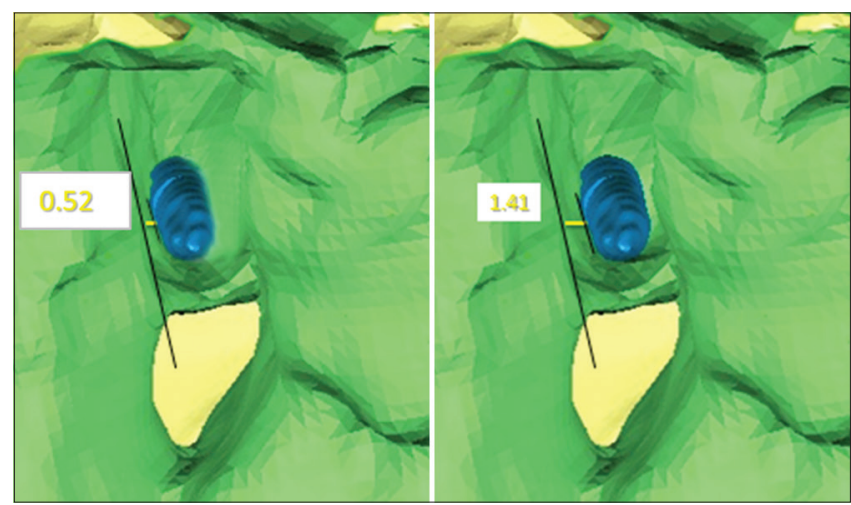

Figure 4: A window of Geomagic software showing the distance between the root of the second premolar and the inserted miniscrews, I: Group A, distance $\leq 0.6 \mathrm{~mm}$, II: Group B, distance $\geq 0.6 \mathrm{~mm}$

\section{Conventional treatment steps}

Separation was performed for first upper and lower molars, followed by banding and bonding for upper and lower arch using American $®$ orthodontic brackets [10] Roth slot 0.022-inch

- Wire sequence for leveling and alignment was; 0.014" Nickel Titanium, 0.016" Nickel Titanium, 0.016 × 0.022 " Nickel Titanium, 0.016 × 0.022 " stainless steel till reaching the working wire $0.017 \times 0.025$ " stainless steel with an average interval 4 weeks between each

- $\quad$ Patients then were referred for first premolars extraction

- For retraction, closing coil springs were installed between the head of the mini-screw and a crimpable hook on the archwire with a force of $100 \mathrm{~g}$ for direct anchorage (Figure 5)

- $\quad$ All patients were treated until the case was fully finished into a class I canine relation with proper leveling and alignment with proper proximal contacts.

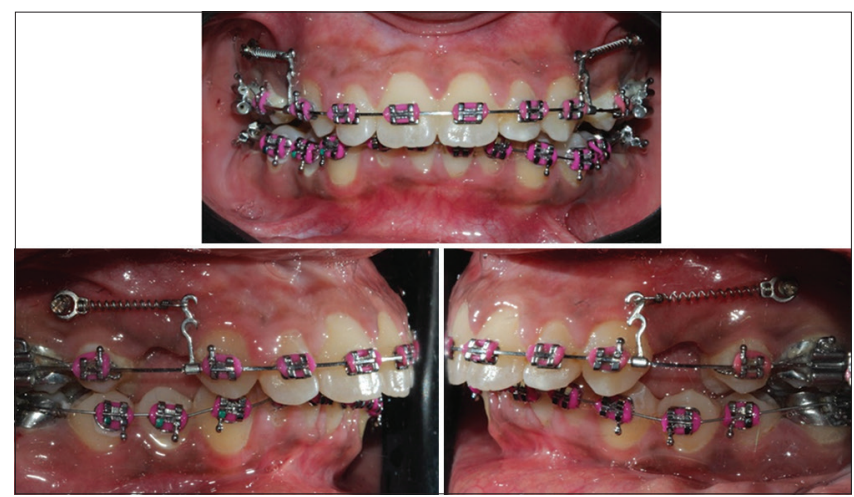

Figure 5: Enmass retraction directly on the mini-screws using closing coils 


\section{Outcome}

- $\quad$ Failure rate was assessed during the period of en mass retraction (8-12 months) [22]

- If the mini-screw was stable in position with no signs of mucosal inflammation or mobility sustaining its function of anchorage, the miniscrew was considered successful

- If the screw showed signs of looseness, mobility, or inflammation that necessitated its removal or relocation, the mini-screw was considered failed (Figure 6).

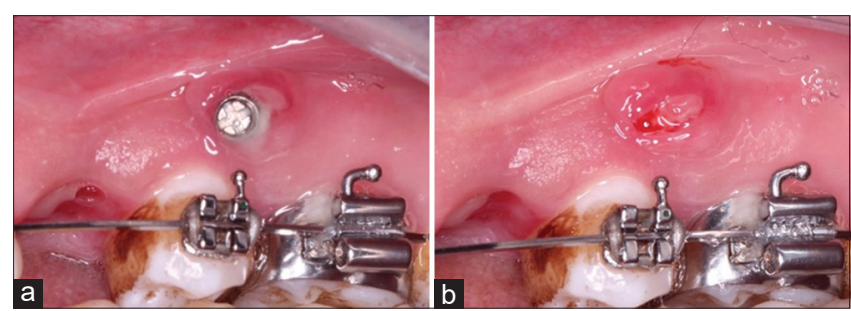

Figure 6: Failed mini-screw, (a) Inflamed tissue surrounding the miniscrew, (b) Mini-screw was removed after failure

\section{Statistical analysis}

- Data were analyzed using IBM Statistical Package for Social Sciences (SPSS) advanced statistics, version 22 (SPSS Inc., Chicago, IL)

- $\quad$ Failure of mini-screws was assessed as binary outcome using Chi-square test

- $\quad p \leq 0.05$ was considered statistically significant. All tests were two tailed.

\section{Results}

The number of the mini-screws in group $A$ was higher in the control than the intervention group with statistical significance difference $(p<0.05)$ (Table 1)

Table 1: Distribution of group A and B among the intervention and control groups (Chi-square test)

\begin{tabular}{|c|c|c|c|c|c|}
\hline \multirow[t]{2}{*}{ Type of treatment } & \multicolumn{2}{|c|}{ Group A } & \multicolumn{2}{|c|}{ Group B } & \multirow[t]{2}{*}{$p$-value } \\
\hline & $n$ & $\%$ & $\mathrm{n}$ & $\%$ & \\
\hline Intervention $(n=42)$ & 3 & 7.14 & 39 & 92.8 & $0.0001^{*}$ \\
\hline $\begin{array}{l}\text { Control }(n=42) \\
\text { p-value }\end{array}$ & $\begin{array}{l}14 \\
0.003\end{array}$ & 33.3 & $\begin{array}{l}28 \\
0.0\end{array}$ & 66.6 & $0.002^{*}$ \\
\hline
\end{tabular}

$\mathrm{N}$ : Number of mini-screws, \%: Percentage, *: Statistical significance.

The failure rate of the mini-screws in both intervention and control group was of a statistically significant difference $(p<0.05)$ as represented in Table 2. The three failed

Table 2: Mini screw failure rate in intervention and control group (Chi-square test)

\begin{tabular}{|c|c|c|c|c|c|}
\hline \multirow[t]{2}{*}{ Failure } & \multicolumn{2}{|c|}{ Successful } & \multicolumn{2}{|c|}{ Failed } & \multirow[t]{2}{*}{$\mathrm{p}$-value } \\
\hline & $\mathrm{n}$ & $\%$ & $\mathrm{n}$ & $\%$ & \\
\hline Intervention & 39 & 92.8 & 3 & 7.14 & $0.0001^{*}$ \\
\hline Control & 35 & 83.3 & 7 & 16.6 & $0.0001^{*}$ \\
\hline$p$-value & 0.214 & & 0. & & \\
\hline
\end{tabular}

mini-screw in the intervention group were inserted in the right side and failed after 1 week (2 mini-screws) and 2 weeks of insertion (1 mini-screw), while the seven failed miniscrews in the control group were inserted in the left sides and they failed at 3 days ( 1 miniscrew), 1 week (4 mini-screws), 1 month (1 mini-screw), and 1.5 months (1 mini-screw) There was no statistically significant difference in failure of mini-screws between both intervention and control groups $(p>0.05)$ as represented in Table 2

Insignificant correlation was found between the root proximity and failure of the mini-screws (Table 3)

Table 3: Correlation between failure and root contact in both intervention and control groups (Pearson's Correlation test)

\begin{tabular}{llll}
\hline $\begin{array}{l}\text { Correlation between root } \\
\text { contact and failure }\end{array}$ & $\mathrm{r}$ & $\mathrm{p}$ & Indication \\
\hline Intervention group & -0.059 & 0.81 & Weak/negative/insignificant \\
Control group & -0.316 & 0.21 & Moderate/negative/insignificant \\
\hline : Pearson's Correlation coefficient & & &
\end{tabular}

r: Pearson's Correlation coefficient.

\section{Discussion}

Smoker patients were not included in the study. Smoking was proven to affect the micro-circulation of the mucosa and gingiva, which in turn may affect the success of the mini-screw [23]. Further, patients with missing posterior teeth in the upper arch were excluded from the study, as that would affect the protocol of extraction of the orthodontic case. Furthermore, the chosen site of the mini-screw insertion might be changed in that case which should be standardized for the entire sample. On the other hand, the $3 \mathrm{D}$ printed guide gained its retention from the occlusal surface of the posterior teeth, thus missing posterior teeth would jeopardize the 3D printed guide's retention and stability during insertion.

Patients with cleft lip and palate were excluded, as they need complex and multi-disciplinary treatment. They usually have disruption of the arch integrity and abnormal eruption pattern which may interfere with selection of the site of mini-screw. Furthermore, the quality of the buccal bone is poorer than usual which may affect the stability of the inserted mini-screw [24]. In addition, patients with facial asymmetry weren't included in the sample. These patients required asymmetric pattern of treatment; either surgical management or asymmetric extraction pattern. Thus, the split-mouth randomized clinical trial could not be fully applied for them [25].

For planning of mini-screw insertion, a preoperative СВСТ was taken, in agreement with previous studies [18], [19], [26], [27], [28], [29], [30]. The CBCT scan was imaged with $90 \mathrm{Kvp}, 6 \mathrm{~mA}, 17.1 \mathrm{mAs}, 2.8 \mathrm{~s}$ with 
an effective radiation dose $21 \mu \mathrm{Sv}$ which considered an ultra-low dose protocol with normal resolution. It was of enough quality to visualize the roots during the planning of the screw position with the least radiation possible [31].

The angle of mini-screw insertion was $40^{\circ}$ with the long axis of the adjacent teeth, which was recommended by Lim et al. [21] to allow for more bone engagement with less risk of root contact. This angle was measured in the planning of both groups; during the insertion in the control group using the conventional technique and during designing the guide for the intervention group.

The distance between the mini-screw and the adjacent root surface shouldn't be $<0.6 \mathrm{~mm}$ as reported by Lee et al. [13] A distance $<0.6 \mathrm{~mm}$ meant that the mini-screw was inside the periodontal space $(0.4 \mathrm{~mm}$ thickness) [32] and the presence of the mini-screw in that distance was reported to provoke inflammatory reaction which may jeopardize the mini-screw's stability [12].

Few of the mini-screws in the intervention group were of group A category, that might be attributed to some dimensional changes in the printed guide allowing some play between the driver and the channel. In addition, more mini-screws of the control group were in group A which might be due to the lack of guidance as the intervention group.

For the failure of the mini-screw, clinical assessment was performed after applying load. It was reported that short-term failure of mini screw was common to occur in the first 3 months with the peak after 2 weeks [33]. Although other studies [9], [34] assessed the failure on longer term (7-12 months), they reported that failure occurred in the first 3 months after loading was of higher incidence, which was consistent to our results where the last mini-screw failed after 1.5 months only.

In terms of failure, the overall failure rate of the all mini-screws inserted in the current study was $11.9 \%$ in agreement of previous studies assessed the failure rate of the mini-screws in general [6], [7], [8], [9], [10], [11], [12], [20], [34], [35], [36], [37], [38], [39], [40]. However, to the best of our knowledge there were no studies assessed the failure rate of the mini-screws inserted by 3D printed guides. In the intervention side, the failure rate was $7.14 \%$. The only mini-screw that failed shortly after its insertion in the intervention group was in the group A. Therefore, that failure might be as a result of the root proximity as reported by previous studies [11], [12], although we found a weak correlation. For the control group, the failure rate was $16.6 \%$. All the failed mini-screws of the control group were also group A. Among the failed mini-screws 5 failed shortly after their insertion which might be due to close proximity to the root as we found moderate correlation between the root proximity and the failure as reported in the literature [11], [12]. The others failed late in the treatment which can be explained that other factors contribute to their failure rather than the root contact alone as mentioned by several studies [9], [35], [36], [41].

\section{Limitations}

The mini-screws were inserted before leveling and alignment which could have resulted in tipping of the adjacent roots before the start of retraction. This could be a confounder to affect the accuracy of the miniscrew failure data. However, as previously explained, the design of the guide and the accuracy of the STL files required clear buccal surfaces of the teeth with no brackets and/or bands.

\section{Conclusions}

Within the limitations of the current study:

- $\quad$ Overall failure rate of mini-screw was $11.9 \%$

- $\quad$ The failure rate was not statistically significant whether inserted by three-dimensional printed guide $(7.14 \%)$ or conventional freehand technique (16.6\%)

Moderate correlation was found between the root proximity and the failure rate of the mini-screws.

\section{References}

1. Nahidh M, Al Azzawi A, Al-Badri S. Understanding anchorage in orthodontics. J Dent Oral Disord. 2019;4(3):1-5.

2. Kyung HM, Park HS, Bae SM, Sung JH, Kim IB. Development of orthodontic micro-implants for intraoral anchorage. J Clin Orthod. 2003;37(6):321-8; quiz 314

PMid:12866214

3. Kravitz N, Kusnoto B. Risks and complications of orthodontic miniscrews. Am J Orthod Dentofac Orthop. 2007;131 Suppl 4:S43-51. https://doi.org/10.1016/j. ajodo.2006.04.027

PMid:17448385

4. Papadopoulos M, Tarawneh F. Skeletal Anchorage in Orthodontic Treatment of Class II Malocclusion: Contemporary Applications of Orthodontic Implants, Miniscrew Implantsand Mini Plates. $1^{\text {st }}$ ed. Amsterdam, Netherlands: Elsevier Ltd.; 2015.

5. Papadopoulos M, Tarawneh F. The use of miniscrew implants for temporary skeletal anchorage in orthodontics: A comprehensive review. Oral Surg Oral Med Oral Pathol Oral Radiol Endod. 2007;103(5):e6-15. https://doi.org/10.1016/j.tripleo.2006.11.022 PMid:17317235

6. Dalessandri D, Salgarello S, Dalessandri M, Lazzaroni E, Piancino M, Paganelli C, et al. Determinants for success rates of temporary anchorage devices in orthodontics: A metaanalysis (n>50). Eur J Orthod. 2014;36(3):303-13. https://doi. org/10.1093/ejo/cjt049 


\section{PMid:23873818}

7. Manni A, Cozzani M, Tamborrino F, de Rinaldis S, Menini A Factors influencing the stability of miniscrews. A retrospective study on 300 miniscrews. Eur J Orthod. 2011;33(4):388-95. https://doi.org/10.1093/ejo/cjq090 PMid:20926556

8. Maino B, Pagin P, Di Blasio A. Success of miniscrews used as anchorage for orthodontic treatment: Analysis of different factors. Prog Orthod. 2012;13(3):202-9. https://doi.org/10.1016/j. pio.2012.04.002

PMid:23260530

9. Aly S, Alyan D, Fayed M, Alhammadi M, Mostafa Y. Success rates and factors associated with failure of temporary anchorage devices: A prospective clinical trial. J Investig Clin Dent. 2018;9(3):e12331. https://doi.org/10.1111/jicd.12331 PMid:29512336

10. Min K, Kim S, Kang K, Cho JH, Lee EH, Chang NY, et al. Root proximity and cortical bone thickness effects on the success rate of orthodontic micro-implants using cone beam computed tomography. Angle Orthod. 2012;82(6):1014-21. https://doi. org/10.2319/091311-593.1

PMid:22417652

11. Gintautaitè G, Gaidytè A. Surgery-related factors affecting the stability of orthodontic mini implants screwed in alveolar process interdental spaces: A systematic literature review. Stomatologija. 2017;19(1):10-8

PMid:29243679

12. Kuroda S, Yamada K, Deguchi $T$, Hashimoto $T$, Kyung $H$, Yamamotod T. Root proximity is a major factor for screw failure in orthodontic anchorage. Am J Orthod Dentofacial Orthop. 2007;131 Suppl 4:68-73. https://doi.org/10.1016/j. ajodo.2006.06.017

PMid:17448389

13. Lee $\mathrm{Y}$, Kim J, Baek S, Kim T, Chang Y. Root and bone response to the proximity of a mini-implant under orthodontic loading. Angle Orthod. 2010;80(3):452-8. https://doi. org/10.2319/070209-369.1 PMid:20050736

14. Baik U, Kook Y, Tanaka O, Kim K. Root contact with miniscrews during mesiodistal movement of the molar. J World Fed Orthod. 2014;3(2):e95-100.

15. Suzuki E, Buranastidporn B. An adjustable surgical guide for miniscrew placement. J Clin Orthod. 2005;39(10):588-90. PMid:16244426

16. Morea C, Dominguez G, Wuo A, Tortamano A. Surgical guide for optimal positioning of mini-implants. J Clin Orthod. 2005;39(5):317-21.

PMid:15961891

17. Cousley RR, Parberry DJ. Surgical stents for accurate miniscrew insertion. J Clin Orthod. 2006;40(7):412-7; quiz 419. PMid:16902252

18. Kim S, Kang J, Choi B, Nelson G. Clinical application of a stereo-lithographic surgical guide for mini-implants. World $J$ Orthod. 2008;9(4):371-82

PMid:19146019

19. Qiu L, Haruyama N, Suzuki S, Yamada D, Obayashi N. Accuracy of orthodontic miniscrew implantation guided by stereolithographic surgical stent based on cone-beam CT-derived 3D images. Angle Orthod. 2012;82(2):284-93. https://doi.org/10.2319/033111-231.1

PMid:21848407

20. Hourfar J, Bister D, Kanavakis G, Lisson J, Ludwig B. Influence of interradicular and palatal placement of orthodontic miniimplants on the success (survival) rate. Head Face Med.
2017;13(1):14. https://doi.org/10.1186/s13005-017-0147-z

PMid:28615027

21. Lim J, Won H, Yoon S. Quantitative evaluation of cortica bone thickness and root proximity at maxillary interradicular sites for orthodontic mini-implant placement. Clin Anat. 2008;21(6):486-91. https://doi.org/10.1002/ca.20671 PMid:18698651

22. Schneider PP, Kim KB, Da Costa Monini A, Dos Santos-Pinto A Gandini LG Jr. Which one closes extraction spaces faster: En masse retraction or two-step retraction? A randomized prospective clinical trial. Angle Orthod. 2019;89(6):855-61. https://doi.org/10.2319/101618-748.1 PMid:31259616

23. Jung B, Liechti T. Prognostic parameters contributing to palatal implant failures: A long-term survival analysis of 239 patients. Clin Oral Implants Res. 2012;23(6):746-50. https://doi. org/10.1111/j.1600-0501.2011.02197.x PMid:21545530

24. Rocha R, Ritter D, Locks A, De Paula L, Santana R. Ideal treatment protocol for cleft lip and palate patient from mixed to permanent dentition. Am J Orthod Dentofacial Orthop. 2012;141(4):S140-8. https://doi.org/10.1016/j. ajodo.2011.03.024 PMid:22449594

25. Thiesen G, Gribel F, Freitas M, Mota P. Facial asymmetry: A current review. Dental Press J Orthod. 2015;20(6):110-25 https://doi.org/10.1590/2177-6709.20.6.110-125.sar PMid:26691977

26. Bae M, Kim J, Park J, Cha J, Kim H. Accuracy of miniscrew surgical guides assessed from cone-beam computed tomography and digital models. Am J Orthod Dentofacial Orthop. 2012;143(6):893-901. https://doi.org/10.1016/j. ajodo.2013.02.018 PMid:23726340

27. Cassetta M, Altieri F, di Giorgio R, Barbato E. Palata orthodontic miniscrew insertion using a CAD-CAM surgical guide: Description of a technique. Int J Oral Maxillofacial Surg. 2018;47(9):1195-8. https://doi.org/10.1016/j.ijom.2018.03.018 PMid:29653870

28. Kim S, Choi Y, Hwang E, Chung K, Kook Y, Nelson G. Surgical positioning of orthodontic mini-implants with guides fabricated on models replicated with cone-beam computed tomography. Am J Orthod Dentofacial Orthop. 2007;131 Suppl 4:S82-9. https://doi.org/10.1016/j.ajodo.2006.01.027 PMid:17448391

29. Liu H, Liu D, Wang G, Wang C, Zhao Z. Accuracy of surgical positioning of orthodontic miniscrews with a computer-aided design and manufacturing template. Am J Orthod Dentofacial Orthop. 2010;137(6):728.e1-10. PMid:20685519

30. Wang $Y, Y u$ J, Lo L, Hsu P, Lin C. Developing customized dental miniscrew surgical template from thermoplastic polymer material using image superimposition, CAD system, and 3D printing. Biomed Res Int. 2017;2017:1906197. https://doi. org/10.1155/2017/1906197

PMid:28280726

31. Ahmed D. Ultra-low-dose versus normal-dose scan protocol of planmeca promax $3 \mathrm{D}$ mid cbct machine in detection of second mesiobuccal root canal in maxillary molars: An ex vivo study. Egypt Dent J. 2019;65(1):221-9.

32. Dean R. The periodontal ligament: Development, anatomy and function. J Oral Health Dent Manag. 2017;16(6):1-7.

33. Moon $C$, Lee $D$, Lee $H$, Im JB. Factors associated with the success rate of orthodontic miniscrews placed in the upper and lower posterior buccal region. Angle Orthod. 2008;78(1):101-6. 
34. Crismani A, Bertl M, Čelar A, Bantleon H, Burstone C. Miniscrews in orthodontic treatment: Review and analysis of published clinical trials. Am J Orthod Dentofacial Orthop. 2010;137(1):108-13. https://doi.org/10.1016/j.ajodo.2008.01.027

PMid:20122438

35. Alharbi $F$, Almuzian $M$, Bearn $D$. Miniscrews failure rate in orthodontics: Systematic review and meta-analysis. Eur J Orthod. 2018;40(5):519-30. https://doi.org/10.1093/ejo/cjx093 PMid:29315365

36. Dalessandri D, Salgarello S, Dalessandri M, Lazzaroni E, Piancino M, Paganelli C, et al. Determinants for success rates of temporary anchorage devices in orthodontics: A metaanalysis (n>50). Eur J Orthod. 2014;36(3):303-13. https://doi. org/10.1093/ejo/cjt049

PMid:23873818

37. Hong S, Kusnoto B, Kim E, Begole E, Hwang H, Lim H. Prognostic factors associated with the success rates of posterior orthodontic miniscrew implants: A subgroup meta-analysis. Korean J Orthod. 2016;46(2):111-26. https://doi.org/10.4041/ kjod.2016.46.2.111

PMid:27019826
38. Papageorgiou S, Zogakis I, Papadopoulos M. Failure rates and associated risk factors of orthodontic miniscrew implants: A metaanalysis. Am J Orthod Dentofacial Orthop. 2012;142(5):577-95 https://doi.org/10.1016/j.ajodo.2012.05.016

PMid:23116500

39. Mohammed $H$, Wafaie K, Rizk M, Almuzian M, Sosly R, Bearn D. Role of anatomical sites and correlated risk factors on the survival of orthodontic miniscrew implants: A systematic review and meta-analysis. Prog Orthod. 2018;19(1):36. https:// doi.org/10.1186/s40510-018-0225-1

PMid:30246217

40. Melo A, Andrighetto A, Hirt S, Bongiolo A, Silva S, Silva M. Risk factors associated with the failure of miniscrews-a ten-year cross sectional study. Braz Oral Res. 2016;30(1):e124. https:// doi.org/10.1590/1807-3107BOR-2016.vol30.0124 PMid:27783770

41. Watanabe H, Deguchi T, Hasegawa M, Ito M, Kim S, TakanoYamamoto T. Orthodontic miniscrew failure rate and root proximity, insertion angle, bone contact length, and bone density. Orthod Craniofac Res. 2013;16(1):44-55. https://doi. org/10.1111/ocr.12003

PMid:23311659 